\title{
Low-Code Platform for Application Development
}

\author{
Khushi Talesra \\ Dept. of Computer Science Engineering \\ R.V. College Of Engineering, Bengaluru
}

\author{
Dr. Nagaraja G.S \\ Dept. of Computer Science Engineering \\ R.V. College Of Engineering, Bengaluru
}

\begin{abstract}
In today's digital world, with a rapid growth in number of companies and ever-changing market requirements, the benefit of low-code solutions can be an important step in providing the technology required to automate the creation and deployment of essential business applications and encourage digital transformation. The interest towards these platforms has significantly augmented in business industry since past few years as more research is conducted towards them. Low code development platforms (LCDPs) provide user friendly visual environments to create software applications with attractive UI, responsive designs, and minimal programming skills.

This paper discusses how low-code platforms can facilitate the building of secure and scalable applications with outstanding features. It discusses the challenges addressed by low-code solutions. Also, the use of Oracle APEX low-code platform in Application development is discussed with respect to building of User Access Audit and control automation application.
\end{abstract}

Keywords: Low code platforms (LCDPs), Oracle Application Express (APEX), Automation, user access audit

\section{INTRODUCTION}

With the rapid acceleration of digital transformation companies are looking for platforms that can facilitate the rapid development and delivery of apps that their business needs with the existing talent. They are looking for quicker, cheaper, and safer ways to meet their software needs without comprising with the quality. In such a scenario low code development platform (LCDPs) have emerged as an assurance for the companies to achieve this.

Low code development platforms (LCDPs) enable the creation of applications with minimal efforts utilizing graphical user interfaces and visual abstraction that automates every step of application development lifecycle. According to Forrester study, today around $84 \%$ of enterprises have turned towards low-code platforms for its ability to reduce the amount of stress on IT resources, lowered cost, increase speed-to-market and better business stakeholder engagement. Thus, with the aim of tackling with the shortage of professional and high skilled developers it allows professionals with no particular programming background to add to the software development process without compromising with the productivity of experienced and skillful developers.

Since past few years, the market for low-code development platforms has been thriving with competitions among multiple sellers to offer the most intuitive and the best development environment for users to fulfil their requirements. Various lowcode platform providers include Mendix, Salesforce, Appian, Google App Maker, OutSystems, Oracle APEX, Microsoft PowerApps etc. Low-code industry is developing at a fast rate and by 2024 , it is forecasted that the development of a low-code application will be accounted for more than $65 \%$ of application development process.

Oracle APEX is a low-code application development platform with a significant number of features. It allows users to visualize, analyze and manage data by creating web applications from an existing database or imported from a spreadsheet. The resulting applications are full-stack web applications comprising HTML/CSS pages and interactive forms consisting of buttons with dynamic actions and processes, JavaScript-powered visualization and logic, and connectors to the external data sources or APIs.

\section{RELATED WORK}

A technical survey about various low-code platforms and their comparative study has been presented by Apurvanand Sahay et al. in [1]. Along with this general architecture of low-code platforms has been discussed. A new approach towards automating business processes using the low-code platform, Aurea BPM has been discussed in [2]. The risk of errors and the time required for creating business applications has been decreased with the use of such platforms. Along with being of great importance in business and IT industry, LCDPs like oracle APEX have offered new opportunities in higher education by making basic application creation an easy task as discussed by Alenka Baggia et al. in [3]. With most business processes being automated today and with the increasing number of employees in companies an automated user access audit system can be significant. The importance of user access review and the best practices to be followed to avoid risks scenarios has been discussed in [5]. The use of artificial intelligence and robotic processes in audit engagement is presented by Chanyuan (Abigail) Zhang [6] but these practices are expensive in terms of employee training, software acquisition, consulting, and software maintenance. To avoid these drawbacks and keeping the importance of audit automation in consideration, this paper proposes a model to build an application for user access review and audit control using low-code platform, oracle APEX. Such a model can help to avoid the errors and reduce the time of auditing when compared with the manual process and will also be a costefficient approach. 


\section{LOW CODE PLATFORMS}

\section{A. Main Components}

The components of any low code platform can be categorized into three tiers: application modeler, server side and its functionalities, and the other external systems that are integrated with low-code platform.

The application modeler is concerned with the developing of the application by providing modelling constructs and a graphical interface. It includes various widgets, drag and drop capabilities, authentication and authorization schemes, data model, business logic flows, connectors and more.

Fig.1. shows part of the application modeler of Oracle APEX. The right pane is used customize the page and page items on it by selecting from drop down menus or writing PL/SQL queries according to the requirement. Security rules can also be changed in the right pane. The middle pane shows the current page organization of how different items are arranged into different regions. The bottom part of middle pane can be used to insert buttons, text field, select list and more. Lastly, the left pane shows your page items like the columns, buttons, tabs in a hierarchal fashion. It also has the option to create dynamic actions, processes, AJAX call-backs and more.

The middle tier consists of compilers, code generators, optimizers. It takes the model of an application which is received from the modeler and then perform operations like code generation and optimization for model management. It also considers the services including API connectors, database systems, the model repositories of a reusable artifacts and collaboration means.

Database servers can be both NoSQL as well as SQL. For example, Oracle APEX has SQL workshops that includes object browser to create database links, tables, synonyms, procedures etc. It also includes SQL commands and SQL scripts to execute SQL and PL/SQL commands. LCDPs also support collaborative activities by supporting software development methodologies like agile, scrum. Modelers can effortlessly visualize the overall application development process, a number of tasks, sprints for various software development methods and deal with changes according to customer requirement.

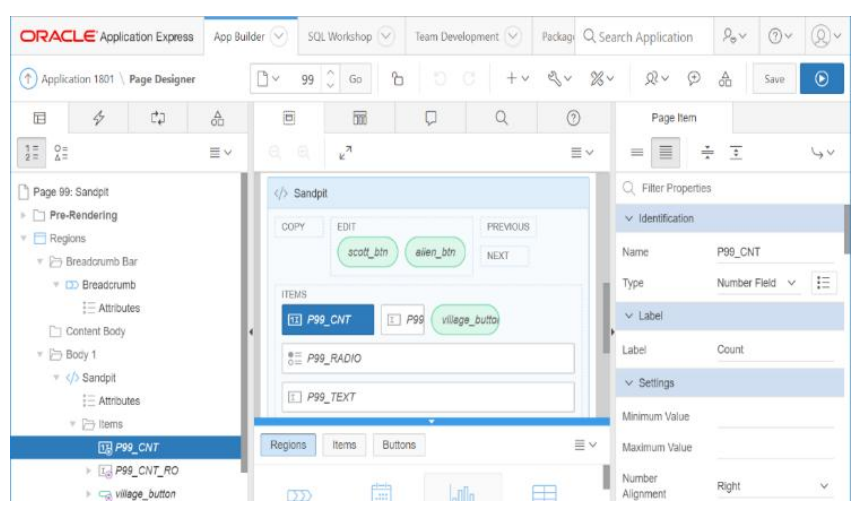

Fig. 1. Oracle APEX Page Designer

\section{B. Pros of Low code platforms}

\section{Greater Productivity:}

As the development time of the app is reduced, more apps can be built in less time leading to higher productivity. Low-code development platforms have brought rapidity to the application building process and have also helped in ensuring that time is no longer a barrier in the process.

Decreased Costs:

As the time required to build apps is reduced, cost is also decreased. These platforms also reduce the number of developers required, reducing hiring costs.

\section{Easy Maintenance:}

Low-code platforms make it possible to quickly change what has been developed already and thus makes maintenance phase easier. Whenever required, changes can be done easily without getting into complex coding.

\section{Involvement of business profiles:}

The gap between IT and business teams is bridged by low-code platforms and both can solve issues that impact the company leading to higher productivity. These platforms provide easy to use interface for application development and thus even business users who will be the final users of the app can work in the development process of the application in collaboration with IT.

\section{Cross platform accessibility:}

The cross-platform accessibility allows users to build applications that can accessed and run on all platforms and devices making it easy to work on any issues. This will also help to improve consumer experiences.

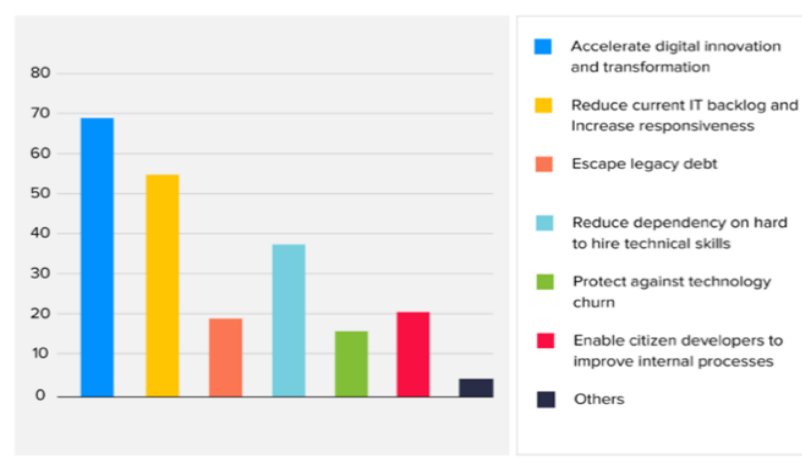

Fig. 2. Main causes for using low-code development platforms

\section{Cons of Low code platforms}

\section{Lack of customization:}

Low code platforms offer limited customization options. This can lead to a problem sometimes when applications need to be very customized and existing modular components cannot provide the required features. In such cases its usage can be limited.

\section{Limited integration options:}

It limits integration options for developers. Some platforms allow you to integrate with a limited list of services. This can be a major challenge for companies with legacy systems. 
Third-part dependence:

One must rely on vendors to mitigate risks or to fix any security issue and changing our schedule for updates to match with that of vendor's. This creates a situation of concern for the companies about lock-in with a vendor.

\section{OVERVIEW AND ANALYSIS OF VARIOUS LOW- CODE PLATFORMS}

\section{Some of the common low-code platforms are:}

\section{Kissflow}

It is a cloud-based platform focused on workflow automation for business processes. Some of its features to support flawless workflows include uncomplicated, simple design that is easy to use interface, handles increased data volume and users easily, real time analytics keeps one aware of the state of each workflow and easy linking with external APIs.

\section{Microsoft PowerApps}

A development platform for mobile and web applications. Supports rapid building of applications through prebuilt templates or start from scratch, drag and drop facility and no coding for basic app development. People with no IT background can also become app builders. Enable extensibility through integration with azure functions. Suitable for mid sizes and large enterprises.

\section{OutSystems}

Low-code development platforms that allows to build both mobile and web applications. Enables development with the help of visual model with rapid productivity. Integration of customized code can be done to create complex apps. Supports develop once and deploy anywhere feature. Preferred by small, mid-sized companies and enterprises. No vendor lock in as it generates apps with a standard architecture.

\section{Mendix}

It is the first low-code development platform to create apps that are intelligent (use machine learning, artificial intelligence and analytics to make predictions and recommendations), contextual (using personal and sensor data apps can be customized for user) and proactive (support push notifications, chatbots). It has cloud native architecture and build apps effortlessly with visual development tools and drag-and-drop facilities. The distinguishing feature of Mendix is the addition of Task Queue in Mendix 9. It allows the app to perform better when tasks take a long time to complete or when there is high volume of transactions. The tasks which take more time to run are performed in the background so that users don't have to wait and can continue working within the app.

\section{Appian}

It is a platform that helps to build workflows and apps at a faster rate with features like visual no-code designer, powerful lowcode tools, beautiful UX and collaborative development. Build once deploy anywhere feature by designing app once and deploying it anywhere. It takes more time for processing larger amounts of data and therefore less suitable for that.

Table 1 shows a comparative analysis of the different low-code platforms discussed above based on different features.

Table 1: Comparative analysis of various low code platforms

\begin{tabular}{|c|c|c|c|c|c|c|}
\hline & Kissflow & $\begin{array}{l}\text { Microsoft } \\
\text { PowerApps }\end{array}$ & OutSystems & Mendix & Appian & Oracle APEX \\
\hline $\begin{array}{l}\text { Visual } \\
\text { Modelling } \\
\text { tools and } \\
\text { user } \\
\text { interface }\end{array}$ & $\begin{array}{l}\text { Visual } \\
\text { modelling } \\
\text { tools like } \\
\text { templates. } \\
\text { Drag and } \\
\text { drop } \\
\text { interface }\end{array}$ & $\begin{array}{l}\text { Pre-built } \\
\text { templates } \\
\text { and various } \\
\text { UI } \\
\text { elements } \\
\text { Component } \\
\text { focused or } \\
\text { model } \\
\text { driven } \\
\text { designing }\end{array}$ & $\begin{array}{l}\text { Visual model } \\
\text { with rapid } \\
\text { productivity } \\
\text { Drag and } \\
\text { drop design }\end{array}$ & $\begin{array}{ll}\text { - Visual } \\
\text { development } \\
\text { tools } \\
\text { Components } \\
\text { are reusable } \\
\text { Drag and drop } \\
\text { facility }\end{array}$ & $\begin{array}{l}\text { Visual no- } \\
\text { code } \\
\text { designer } \\
\text { Drag-and- } \\
\text { drop } \\
\text { interfaces. }\end{array}$ & $\begin{array}{l}\text { - Visual code } \\
\text { developmen } \\
\text { - User } \\
\text { friendly } \\
\text { interface }\end{array}$ \\
\hline Open source & No & No & No & Yes & No & Yes \\
\hline $\begin{array}{l}\text { Built in } \\
\text { workflows }\end{array}$ & Present & Absent & Absent & Absent & Absent & Absent \\
\hline $\begin{array}{l}\text { Learning } \\
\text { curve }\end{array}$ & Easy to learn & $\begin{array}{l}\text { Pretty steep } \\
\text { learning curve }\end{array}$ & $\begin{array}{l}\text { Easy to learn for } \\
\text { developers and } \\
\text { business analysts }\end{array}$ & $\begin{array}{ll}\text { - } & \begin{array}{l}\text { Need } \\
\text { experienced } \\
\text { programmers } \\
\text { and developers }\end{array} \\
\text { - } & \begin{array}{l}\text { No direct tech } \\
\text { support }\end{array} \\
\end{array}$ & $\begin{array}{l}\text { - Not easy to } \\
\text { learn } \\
\text { Training } \\
\text { tutorials can } \\
\text { be made } \\
\text { better }\end{array}$ & Easy to learn \\
\hline $\begin{array}{l}\text { Supported } \\
\text { databases }\end{array}$ & SQL & $\begin{array}{l}\text { Azure, SQL } \\
\text { server, } \\
\text { OneDrive, } \\
\text { Salesforce }\end{array}$ & $\begin{array}{l}\text { Database SQL } \\
\text { server, SQL } \\
\text { Azure, Oracle } \\
\text { MySQL, IBM, } \\
\text { SAP }\end{array}$ & $\begin{array}{l}\text { SQL, Oracle } \\
\text { Database, IBM, } \\
\text { MariaDB, MySQL }\end{array}$ & $\begin{array}{l}\text { Amazon, SQL } \\
\text { Server, IBM }\end{array}$ & Oracle database \\
\hline $\begin{array}{l}\text { Cost and } \\
\text { free trial }\end{array}$ & $\begin{array}{l}\text { Starting from } \\
\text { \$9/user/month } \\
\text { Depending on } \\
\text { the } \\
\text { subscription } \\
\text { Free trial } \\
\text { available }\end{array}$ & $\begin{array}{l}\$ 7-\$ 40 \\
\text { /user/month } \\
\text { No free trial } \\
\text { available }\end{array}$ & $\begin{array}{l}\text { Starts at } \\
\$ 4000 / \text { month } \\
\text { Costly for one } \\
\text { application } \\
\text { Free trial } \\
\text { available }\end{array}$ & $\begin{array}{l}\text { Starts from } \\
\$ 1875 / \text { month } \\
\text { Free trial available }\end{array}$ & $\begin{array}{l}\text { Starts at } \\
\text { \$90/month } \\
\text { Free trial } \\
\text { available }\end{array}$ & $\begin{array}{l}\text { No per user or } \\
\text { application cost. } \\
\text { License to } \\
\text { peripheral } \\
\text { components } \\
\text { needed } \\
\text { Free trial } \\
\text { available }\end{array}$ \\
\hline Deployment & Cloud & Cloud & Cloud, Saas, Web & $\begin{array}{l}\text { Private and public } \\
\text { cloud, On premise }\end{array}$ & $\begin{array}{l}\text { On premise, } \\
\text { Saas }\end{array}$ & $\begin{array}{l}\text { Oracle database } \\
\text { cloud service, } \\
\text { private and } \\
\text { public clouds, } \\
\text { on premise }\end{array}$ \\
\hline
\end{tabular}




\section{ORACLE APPLICATION EXPRESS (APEX)}

The Oracle Application Express (APEX) is also a low-code development platform that enables us to create database-centric enterprise web applications which are scalable, secure and can be deployed anywhere. Using a web browser and finite programming experience programs can be developed easily. The various built-in features such as interactive reports, navigational controls, other form handlers, interactive grid, various kinds of charts, calendars help to facilitate the application development process. It provides user with following remarkable advantages: faster application development and its subsequent delivery, easy to develop and deploy applications, secure and portable, eliminates the complexity of application development at all layers.

\section{A. Architecture of Oracle APEX}

It follows a 3-tier architecture-

- Client tier which is the browser

- Middle tier is formed by the APEX application engine

- The data tier consists of back-end databases and REST.

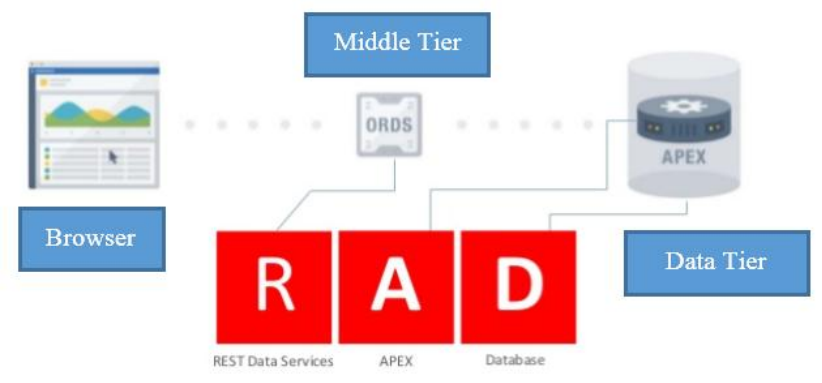

Fig. 3. Oracle APEX architecture

From the browser, web request is sent to the ORDS (oracle REST data services) and from there they are sent to Oracle database. In the database, the submitted request is processed by Oracle APEX. Database involves the processing of request, data manipulation and execution of business logic. After the processing has been done result is sent again to the browser through ORDS.

Oracle RAD Stack:

It consists of three main core components:

\section{REST data services}

Enables developers to declaratively create REST Data Access APIs from oracle APEX. Facilitates the integration and handles the micro services.

Allows to create APIs easily by REST-enabling database objects such as tables, views, or PL/SQL.

\section{APEX}

Low code application development platform to build apps. Used by IT professionals, business professionals and developers. Provides intuitive developer experience, intelligent wizards, easy page design and simplified development.

\section{Database}

Oracle Database - the most integrated, complete, and reliable database solution for any scale deployment.

\section{B. Distinguishing Features of Oracle APEX}

\section{Faceted Search:}

For quickly searching by narrowing down search results and filtering data. It creates a multi-dimensional navigation UI automatically depending upon the data patterns in tables.

\section{Turn data into information:}

The interactive report component allows users to make changes to data they see like filtering of columns, sorting or grouping data, viewing it in entirely new ways using charts and then saving these customizations.

\section{Edit data effortlessly:}

Interactive grids provide all the features for editing data like sorting. aggregates, row and column selection, multiple filters and more.

\section{Charting capabilities:}

Responsive and highly customizable charts are provided with a number of ways to analyse data including line, bar, pie, funnel and more.

\section{RESTful web services:}

The RESTful Web Service Wizard in the SQL Workshop area of Oracle Application Express allows the user to implement a RESTful Web Service. Once created it can be called using Uniform Resource Identifier (URI). With the services of RESTEnabled SQL from the Oracle REST Data Services, we have access to the data and perform SQL based queries on remote Oracle databases over REST and HTTP.

User Interface:

Highly customizable and responsive UI can be developed using HTML, CSS, or Java Script.

\section{Security:}

Builds highly secure apps through authorization and authentication schemes. It also allows users to write customised authorisation and authentication schemes in PL/SQL.

\section{Session Management:}

The management of server-side session is an important oracle APEX part. Session control by inherent timeouts for the session, it automatically ends when the user is absent for too long.

\section{USER ACCESS AUDIT AND CONTROL AUTOMATION APPLICATION USING ORACLE APEX}

\section{A. Objective of Automation}

Most business processes today are automated, and companies continue to invest towards this automation and increase it with the help of advanced technologies. With the increasing number 
of IT systems and employees, the overall process of establishing user the access review to keep a check on accesses of the employees has become augmented with complication and is time-consuming. Companies often find it demanding to meet their audit requirements, with the present error prone system which is a manual review process and are searching for a better solution to deliver a quality access review that can be executed in a timely manner. The use of traditional spreadsheets in current system of user access audit and control involves a lot of manual work and there is a need to automate this process by designing application that are scalable and more secure with better features.

Using Oracle APEX, application for automating the audit process can be built easily. Modern and intuitive UI can be provided using various features of APEX such as interactive forms and reports, interactive grid, navigation controls. The various shortcomings of manual access review process can be overcome by building a customizable and responsive application using Oracle APEX. Automated user access audit and control also empowers an increase in the scale of audit since the process will no longer be limited by the processing power of human beings. Fig.: 4, shows the high-level design of the system developed.

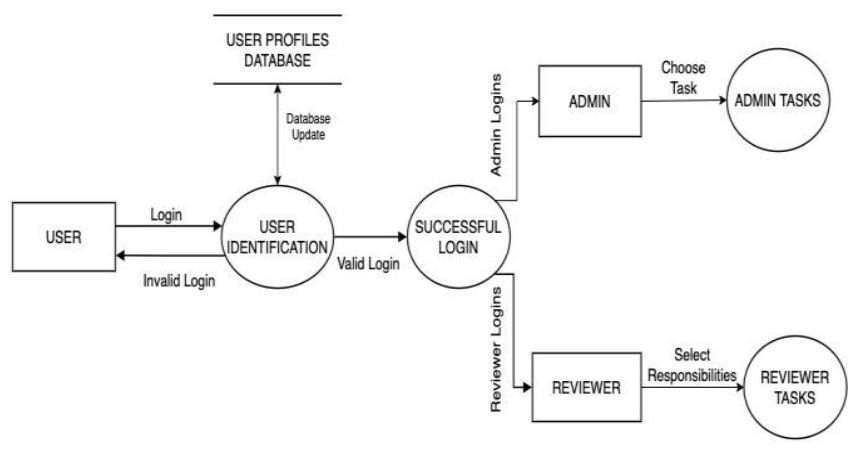

Fig. 4. High-Level design of the system

\section{B. Features Provided}

- App Development IDE which is a web browser and hence it does not need a client software.

- Each step of manual process has been automated by mapping it to a certain step in application.

- Reviewers can log-in, revoke and check the accesses directly.

- Thus, error free and automated review system can be provided.

\section{Basic steps in creation process}

i) Data Modelling: A simple data model of the system being created is further developed by making entities, defining various type of relationships between them and also the constraints and dependencies. It gives an overview of the application being developed. ii) User Interface Definition:

Interactive Forms, Interactive grids, form, classical report, report with form were used to develop different pages of the application which formed the application view. These reports differ in the extent and way in which the end customers can customize the overall look of the available data by adding features like sorting, searching, highlighting, filtering, column selection, and different data manipulations. A table created in object browser or through customized PL/SQL query was used to form the content of the page.

The editing of the page was done in page designer where various items such as buttons, text fields, select list were also added according to the requirement from the middle pane. For submission of page, buttons were created and dynamic actions were attached to them and under database action SQL insert/delete/update action can be selected depending on the need.

The columns of the page are displayed in the left pane of page designer and each was customized by choosing different types from right pane like hidden, text field, number field and more. For selection of access to be revoked checkboxes were used.

There can be two different users of this application. Either an admin can log-in or a reviewer. To make certain navigation menu fields visible only for Admin, customized PL/SQL query was written for that field under navigation menu in shared components.

Admin can check for new responsibilities and missing column values and update them using the edit option. Any changes made are updated in database by creating processes or dynamic actions on buttons. A page creation is a single unit of logic in PL/SQL that works when a particular event occurs, such as submitting or loading a page.

Reviewer can review the accesses, select the accesses to be revoked and request for their revoke.

iii) Placement of other external services by using thirdparty APIs: The background process of revoking the accesses from database and sending emails to notify the reviewers at the end was done by invoking APIs.

iv) Application Development: After completing the designing part the developed application is previewed and deployed.

\section{CONCLUSION}

Low-code platforms are a major step towards building apps rapidly, efficiently, and creatively. With more improvements and upgradations in these platforms, the apps produced will get better each day. Companies will soon have production ready apps created at a much faster pace. Major use cases of low code platforms have been seen in applications related to business process managing and database managing. 
An application of these platforms has been shown in the automation of user access auditing process. The earlier manual process was time consuming and prone to errors. With the help of created application these drawbacks were overcome, and an error free and automated review system was built. The app built was secure as apex provides authentication schemes for establishing the identity of the user, reliable, good performance as each request took minimum time to process and had a userfriendly interface. Also, the overall cost for development and deployment of application was minimum. Web application development with oracle APEX gives users a window of options to build applications with ease and world class features with nominal skills and knowledge in programming. They also get an experience of working in cloud.

Along with the plethora of benefits of using low-code platform for application development, it also has some limitations as mentioned like lack of customization, vendor dependence, limited integration options. It is important to keep in mind that low-code platforms cannot eliminate the need of traditional development skills entirely. If the platform is not able to fulfil the business requirements with tools available, the only thing that can help is the programming skills of developers.

\section{REFERENCES}

[1] Apurvanand Sahay, Arsene Indamutsa, Davide Di Rusico, Alfonso Pierantonoi, 2020, "Supporting the understanding and comparison of low-code development platforms", 46th Euromicro Conference on Software Engineering and Advanced Applications (SEAA), virtual event.

[2] Robert Waszkowski, 2019, "Low-code platform for automating business processes in manufacturing”, IFACPapersOnLine Volume 52, Iissue 10.

[3] Alenka Baggia, Robert Leskovar,Blaž Rodič, 2019, "Low code programming with oracle APEX offers new opportunities in higher education", third International Scientific Conference ITEMA Recent Advances in Information Technology, Tourism, Economics, Management and Agriculture

[4] Raquel Sanchis, Óscar García-Perales, Francisco Fraile and Raul Poler, 2019, "Low-Code as Enabler of Digital Transformation in Manufacturing Industry", Applied Sciences 10(1):12 DOI:10.3390/app10010012.
[5] Sundaresan Ramaseshan, 2019, "Effective User Access Reviews”, ISACA Journal, Volume 4.

[6] Chanyuan (Abigail) Zhang, 2019, "Intelligent Process Automation in Audit", Journal of Emerging Technologies in Accounting, Volume 16, Issue 2

[7] Michael G. Alles, Alexandeer Kogan, Miklos A. Vasarhelyi, 2008, “Audit Automation for Implementing Continuous Auditing: Principles and Problems", Rutgers Business School, Department of Accounting, Business Ethics \& Information Systems.

[8] Hany F. Atlam, Muhammad Ajmal Azad, Madini O. Alassafi , Abdulrahman A. Alshdadi and Ahmed Alenezi, 2020, "Risk-Based Access Control Model: A Systematic Literature Review", Future Internet Journal, Volume 12, Issue 6.

[9] Amandeep Singh, Pardeep Mittal, and Neetu Jha, 2013, "Foss: A challenge to proprietary software", IJCST, Vol. 4 , Issue 3.

[10] Sowmya S, Deepika Dash, 2020 "Benefits and Limitations of Appian- Business Process Model (BPM) Tool: Systematic Literature Review and Research Survey", International Research Journal of Engineering and Technology (IRJET), Volume: 07 Issue: 06.

\section{AUTHOR'S PROFILE}

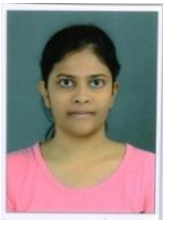

Khushi Talesra, an undergraduate student, pursuing her engineering course in computer science and engineering at R.V. College of Engineering, Bengaluru, India in the academic year 2020-21. Her area of interest involves machine learning, website development, java developer and database programmer.

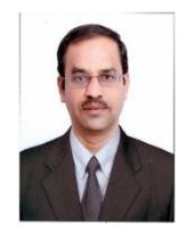

Dr. Nagaraja G.S is currently working as Professor and Associate Dean in the department of Computer Science and Engineering at R.V. College of Engineering, Bengaluru, India. He has publications in various international and national Journals and Conferences. His area of interest involves Computer Networks \& Management, Multimedia Communications, Computer Architecture, Protocol Design. 